Pacific Journal of Mathematic 


\title{
THE MAXIMAL SET OF CONSTANT WIDTH IN A LATTICE
}

\section{G. T. SAlleE}

\begin{abstract}
A new construction for sets of constant width is employed to determine the largest such set which will fit inside a square lattice.
\end{abstract}

A set $W$ in $E^{2}$ is said to have constant width $\lambda($ denoted $\omega(W)=\lambda)$ if the distance between each pair of parallel supporting lines of $W$ is $\lambda$. If $x \in$ bd $W$ we will denote all points opposite $x$ (that is, at a distance $\lambda$ from $x$ ) in $W$ by $0(x)$.

In what follows we will be most concerned with Reuleaux polygons, which are sets of constant width $\lambda$ whose boundaries consist of an odd number of arcs of radius $\lambda$ centered at other boundary points (see [2], p. 128, for a more complete description).

We say a set $S$ avoids another set $X$ if int $S \cap X=\varnothing$.

THEOREM 1. Let $L$ be a square planar unit lattice. Then the unique set of maximal constant width which avoids $L$ is a Reuleaux triangle $T$ having width $\omega(T)>1.545$. An axis of symmetry of $T$ parallels one of the major axex of $L$ and is midway between two parallel rows of the lattice.

The proof depends upon a variational method for altering Reuleaux polygons which will be described in $\S 2$. A useful lemma is also proved there. In $\S 3$ the proof of the theorem is given, while various generalizations are discussed in $\S 4$.

The construction described in the next section was also found independently by Mr. Dale Peterson.

2. Variants of sets of constant width. Let $P$ be a set of constant width $\lambda$ and $p_{0}$ a point near $P$ but exterior to it. Suppose that $q$ and $r$ are the two points on the boundary of $P$ which are at a distance $\lambda$ from $p_{0}$. Let $Q$ be the convex set whose boundary is following: the shorter arc of the circle $C\left(p_{0}, \lambda\right)$ [the circle of radius $\lambda$ centered at $p_{0}$ ] between $q$ and $r$, the boundary of $P$ from $r$ to $q^{\prime}$ (a point opposite $q$ ), an arc of $C(q, \lambda)$ between $q^{\prime}$ and $p_{0}$, an arc of $C(r, \lambda)$ between $p_{0}$ and $r^{\prime}$, and the boundary of $P$ from $r^{\prime}$ to $q$ [see Figure 1]. We call $Q$ the $p_{0}$-variant of $P$. It is easy to see that $Q$ is a set of constant width $\lambda$. In order for the construction to work $p_{0}$ must be close enough to $P$ so that the boundary arc of $P$ between $q$ and 
$r$ on the side nearer $p_{0}$ contains two opposite points. It is also possible to determine the variant by prescribing the two points $q$ and $r$. When this is done, we will refer to $Q$ as the $(q, r)$-variant of $P$.

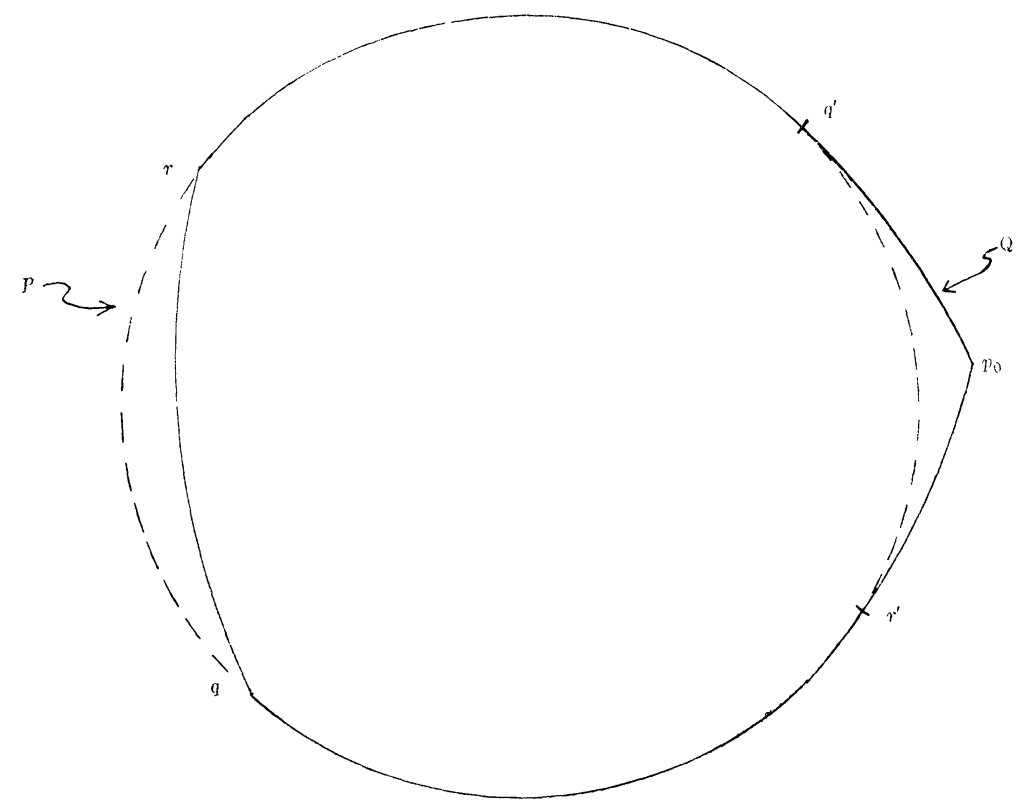

FIgURE 1.

This method gives a way of easily constructing sets of constant width which seems to be new. In particular, applying this method to the unit circle leads to a new class of sets of constant width. A similar construction may be carried out in $d$-dimensional space, and this process will be explored more fully in another paper [4].

The following lemma is more general than necessary, but may be useful for other problems of this nature.

We will say a family of sets in the plane is locally finite if every bounded set meets only a finite number of them.

LEMma 1. Let $\left\{X_{\alpha}: \alpha \in A\right\}$ be a locally finite family of convex sets in the plane and let $X=\cup\left\{X_{\alpha}: \alpha \in A\right\}$. If a set $P$ of maximal constant width avoiding $X$ exists, then it is a Reuleaux polygon with property $\left(^{*}\right)$ : each of the open (curvilinear) edges of $P$ contains at least one point of $X$.

Proof. Suppose $K$ is a set of maximal constant width $\lambda$ which avoids $X$. We shall assume that it is not as described and show that there exists another set having a greater constant width which also avoids $X$. First we will show that for maximality $K$ is a Reuleaux polygon and then that it has property $(*)$. 
Choose $y_{1}$ in bd $K$ and $y_{2}$ in bd $K$ counter-clockwise as far as possible from $y_{1}$ but so that the $\left(y_{1}, y_{2}\right)$-variant of $K$ avoids $X$. Call this variant $K_{1}$. It is not hard to see that $K_{1}=K$ if and only if $K$ is a Reuleaux triangle or else $y_{1}$ and $y_{2}$ are opposite some common point and the set of points opposite $y_{2}, 0\left(y_{2}\right)$, meets $X$. In a similar fashion choose $y_{3}$ in bd $K_{1}$ counterclockwise as far as possible from $y_{2}$ so that the $\left(y_{2}, y_{3}\right)$-variant of $K_{1}$ avoids $X$. Continue in this fashion.

After a finite number of steps this process will lead to a Reuleaux polygon avoiding $X$. For the $y_{i}$ are determined either by one of the $X_{\alpha}$ or else by the fact that two adjacent $y_{j}$ are a distance $\lambda$ apart. Since the $X_{\alpha}$ are locally finite, each of these cases can occur only a finite number of times as the $y_{i}$ get further around bd $K$ from $y_{1}$. The assertion follows.

We have now constructed a Reuleaux polygon $P$ of the same width as $K$ which also avoids $X$. Note that if $K$ itself were not a Reuleaux polygon satisfying $\left({ }^{*}\right)$, it is possible to modify the construction of $P$ slightly (by not choosing the $y_{i}$ to be at a maximal distance in some suitable step) so that $P$ is a Reuleaux polygon, but does not satisfy $\left.{ }^{*}\right)$. We now show that such a $P$ does not have maximal width, contrary to our initial assumption.

In fact, we will construct a sequence of Reuleaux polygons $P_{0}, \cdots$, $P_{m}$ such that $P_{0}=P, P_{i+1}$ is a variant of $P_{i}$ and $P_{i+1}$ has fewer closed edges than $P_{i}$ which contain a point of $X$. Since all the $P_{i}$ will have the same number of edges, the process will produce a Reuleaux polygon $P_{m}$ disjoint from $X$. Then a larger homothet of $P_{m}$ will avoid $X$ contrary to the assumption that $K$ was maximal.

Suppose that $P_{0}$ has vertices $v_{0}, v_{1}, \cdots, v_{2 n}$ and suppose that the open edge $\left(v_{2 n}, v_{0}\right)$ contains no point of $X$, but that $v_{0}$ or $v_{2 n}$ may belong to $X$. Let $v_{n}^{\prime}$ be a point on the arc between $v_{n}$ and $v_{n+1}$, and let $P_{1}$ be the $\left(v_{n-1}, v_{n}^{\prime}\right)$ variant of $P_{0}$. The vertices of $P_{1}$ are

$$
v_{0}, \cdots, v_{n-1}, v_{n}^{\prime}, v_{n+1}, \cdots, v_{2 n-1}, v_{2 n}^{\prime} .
$$

If $v_{n}^{\prime}$ is close enough to $v_{n}, P_{1}$ will avoid $X$ and in particular the halfopen edge $\left[v_{2 n}^{\prime}, v\right]$ contains no points of $X$. Now choose $v_{n}^{\prime \prime}$ on the arc of $P_{1}$ between $v_{n-1}$ and $v_{n}^{\prime}$ and $P_{2}$ be the $\left(v_{n}^{\prime \prime}, v_{n+1}\right)$ variant of $P_{1}$. The other new vertex of $P_{2}$ will be $v_{0}^{\prime}$, near $v_{0}$. If $v_{n}^{\prime \prime}$ is sufficiently close to $v_{n}^{\prime}, P_{2}$ will also avoid $X$ and the closed edge $\left[v_{2 n}^{\prime}, v_{0}^{\prime}\right]$ will contain no point of $X$.

Note moreover that in the obvious correspondence between $P_{0}$ and $P_{2}$, every closed edge of $P_{2}$ containing a point of $X$ corresponds to a closed edge of $P_{0}$ containing a point of $X$. In addition, we may repeat the above construction on the two open edges of $P_{2},\left(v_{n-1}, v_{n}^{\prime \prime}\right)$ and $\left(v_{n}^{\prime \prime}, v_{n+1}\right)$ to produce Reuleaux polygons with at least two open edges 
and more closed edges avoiding $X$.

Continuing the process through at most $2 n$ steps will lead to a Reuleaux polygon of width $\lambda$ disjoint from $X$. By our earlier remarks this completes the proof.

\section{Proof of theorem. The following lemma is needed.}

Lemma 2. Let $L$ be a planar lattice and $K$ a strictly convex set (its boundary contains no line segment) avoiding $L$. Then the boundary of $K$ contains at most four points of $L$.

Proof. Let $Z=K \cap L$. Since $K$ is strictly convex, $Z$ contains only two points in any one direction and these two points have no point of $L$ between them.

Coordinatize the plane (not necessarily with perpendicular axes) so that $L$ corresponds to the integer points of the coordinatization, so that every point of $Z$ lies in the upper half plane, and so that the points $(0,0)$ and $(1,0)$ belong to $Z$. Now suppose $(k, n) \in Z$ for some $n \geqq 3$. Then taking a suitable convex combination of the three points $(0,0),(1,0)$ and $(k, n)$ which all lie on bd $K$ shows that $(m, 1) \in \operatorname{int} K$, where $m=[k / n]+1([x]$ being the greatest integer in $x)$. Then $K$ does not avoid $L$ contrary to hypothesis. Hence every point of $Z$ has $y$-coordinate 0 or 1 . Since no more than two points of $Z$ can be in either of the rows, the assertion is proved.

We can now prove the theorem. By the Blaschke Selection Theorem it is clear that a set of maximal constant width avoiding $X$ exists. Since every set of constant width is strictly convex, and since every lattice is locally finite, the results of Lemmas 1 and 2 imply that the maximal width $\lambda$ is attained by a Reuleaux triangle $T$. It only remains to establish the orientation of $T$.

By Lemma 1, each of the three edges of $T$ contains a lattice point of $L$ and it is clear that they must belong to a unit square of $L$. So suppose $a \equiv(0,1), b \equiv(1,1)$ and $c \equiv(1,0)$ belong to $T$. We wish to show $d \equiv(0,0)$ also belongs to $T$. If $T \cap L$ consists of exactly three points, it follows from Lemma 1 that there is one vertex between each pair of lattice points. Let these vertices be $a^{\prime}, b^{\prime}$, and $c^{\prime}$ where $a^{\prime}$ is opposite $a$, etc.

Suppose $x\left(c^{\prime}\right)$ [the $x$-coordinate of $\left.c^{\prime}\right]>1 / 2$. Rotate $T$ a small distance counter-clockwise to $T^{*}$ so that $T^{*}$ still contains $a$ and $b$ on its boundary. If the rotation is small enough, $d \notin T^{*}$ and the distance between $c$ and $c^{\prime}$ is increased (this latter statement is proved in [1] $\S 2$ where it is shown that the curve $R(x ; l ; \lambda)$ defined there is strictly convex). Then it is clear that a larger homothet of $T^{*}$ will avoid 
$L$ contrary to the choice of $T$. In a similar way we see that the $y$ coordinate of $a^{\prime} \leqq 1 / 2$.

Now if $d \notin T$ either $c^{\prime} d>\lambda$ or $a^{\prime} d>\lambda$. If $c^{\prime} d>c^{\prime} c=\lambda$ then $x\left(c^{\prime}\right)>1 / 2$ in contradiction to what was proved in the last paragraph. We arrive at a similar contradiction by assuming $a^{\prime} d>\lambda$. Hence $d \in T$.

Hence two lattice points are opposite the same vertex of $T$ and thus are equidistant from it. Without loss of generality, suppose $c$ and $d$ are both opposite $c^{\prime}$. Then $x\left(c^{\prime}\right)=1 / 2$ and $T$ is as described in the theorem.

We may compute $\omega=\omega(T)$ as follows. If $T$ is in the orientation just described, and we let

$$
\alpha=y\left(c^{\prime}\right), \beta=y\left(a^{\prime}\right)=y\left(b^{\prime}\right), x\left(a^{\prime}\right)=\frac{1}{2}+\gamma, x\left(b^{\prime}\right)=\frac{1}{2}-\gamma,
$$

we see:

$$
\begin{gathered}
\gamma=\omega / 2 \\
\omega^{2}=1 / 4+\alpha^{2} \\
\beta=\alpha-\sqrt{3} \omega / 2 \\
\left(\frac{1}{2}+\frac{\omega}{2}\right)^{2}+(1-\beta)^{2}=\omega^{2} .
\end{gathered}
$$

Untangling (2), (3) and (4), we obtain:

$$
2 \omega^{4}+\omega^{3}(2 \sqrt{3}-1)+\omega^{2}(-2-\sqrt{3})+\omega(-1-3 \sqrt{ } \overline{3})-2=0 .
$$

Solving (5) leads to the stated value for $\omega(T)$.

It is clear that the techniques used in proving this theorem can be extended to other similar problems. In particular, if $L$ is any planar lattice the set of maximal constant width is again a Reuleaux triangle. In general, Lemma 1 ensures that the maximal figure is a Reuleaux polygon and makes it fairly easy to determine the number of sides, but it is more difficult to determine the exact orientation.

4. Remarks. Let $\mathscr{C}$ be any 2-dimensional Minkowski space with unit ball $S$. We may define $W$ to be a set of constant width $\lambda$ relative to $S$ if $\omega(W, u)=\lambda \omega(S, u)$ for any direction $u$. In analogy to the Euclidean case, we say $R$ is a relative Reuleaux polygon if $R$ is of constant relative width and is the intersection of a finite number of (properly chosen) translates of $\lambda S$.

With only slight changes, the proof of Lemma 1 may be seen to be valid in $\mathscr{C}$ (where, of course, an "are of radius $\lambda$ " is an are of $\lambda S$, etc.). However, sets of constant width relative to $S$ only satisfy 
the hypotheses of Lemma 2 if $\mathscr{C l}$ is rotund-that is, if $S$ is strictly convex.

So we have, in fact, proved the following:

Lemma 3. Let $\left\{X_{\alpha}: \alpha \in A\right\}$ be a locally finite family of convex sets in any 2-dimensional Minkowski space and let

$$
X=\cup\left\{X_{\alpha}: \alpha \in A\right\} \text {. }
$$

Every set of maximal constant relative width avoiding $X$ is a relative Reuleaux polygon with property $\left({ }^{*}\right)$.

THEOREM 2. Let $L$ be a planar lattice in a rotund, 2-dimensional Minkowski space. Every set of maximal constant width avoiding $L$ is a relative Reuleaux triangle with property (*).

The author wishes to thank G. D. Chakerian for calling this problem to his attention and for interesting discussions. It seems to have originally appeared as a problem in the American Math. Monthly [3]. The author also wishes to thank the referee for his suggestion strengthening the statement of Lemma 1.

\section{REFERENCES}

1. G. D. Chakerian and G. T. Sallee, An intersection theorem for sets of constant width (to appear in Duke Math. J.)

2. H. G. Eggleston, Convexity, Cambridge Univ. Press, Cambridge, 1958.

3. J. Hammer, Problem 5368, Amer. Math. Monthly 73 (1966).

4. G. T. Sallee, Reuleaux polytopes (to appear).

Received April 30, 1968.

UNIVERSITY OF CALIFORNIA AT DAVIS 


\section{PACIFIC JOURNAL OF MATHEMATICS}

\section{EDITORS}

\author{
H. ROYDEN \\ Stanford University \\ Stanford, California \\ R. R Phelps \\ University of Washington \\ Seattle, Washington 98105
}

\author{
J. Dugundui \\ Department of Mathematics \\ University of Southern California \\ Los Angeles, California 90007
}

RICHARD ARENS

University of California

Los Angeles, California 90024

\section{ASSOCIATE EDITORS}
E. F. BeCKENBACH
B. H. NeUmanN
F. WolF
K. YosidA

\section{SUPPORTING INSTITUTIONS}

\author{
UNIVERSITY OF BRITISH COLUMBIA \\ CALIFORNIA INSTITUTE OF TECHNOLOGY \\ UNIVERSITY OF CALIFORNIA \\ MONTANA STATE UNIVERSITY \\ UNIVERSITY OF NEVADA \\ NEW MEXICO STATE UNIVERSITY \\ OREGON STATE UNIVERSITY \\ UNIVERSITY OF OREGON \\ OSAKA UNIVERSITY \\ UNIVERSITY OF SOUTHERN CALIFORNIA
}

\author{
STANFORD UNIVERSITY \\ UNIVERSITY OF TOKYO \\ UNIVERSITY OF UTAH \\ WASHINGTON STATE UNIVERSITY \\ UNIVERSITY OF WASHINGTON

$* * * * *$
AMERICAN MATHEMATICAL SOCIETY
CHEVRON RESEARCH CORPORATION
TRW SYSTEMS
NAVAL WEAPONS CENTER

The Supporting Institutions listed above contribute to the cost of publication of this Journal, but they are not owners or publishers and have no responsibility for its content or policies.

Mathematical papers intended for publication in the Pacific Journal of Mathematics should be in typed form or offset-reproduced, double spaced with large margins. Underline Greek letters in red, German in green, and script in blue. The first paragraph or two must be capable of being used separately as a synopsis of the entire paper. It should not contain references to the bibliography. Manuscripts, in duplicate if possible, may be sent to any one of the four editors. Please classify according to the scheme of Math. Rev. 36, 1539-1546. All other communications to the editors should be addressed to the managing editor, Richard Arens, University of California, Los Angeles, California, 90024.

50 reprints are provided free for each article; additional copies may be obtained at cost in multiples of 50 .

The Pacific Journal of Mathematics is published monthly. Effective with Volume 16 the price per volume (3 numbers) is $\$ 8.00$; single issues, $\$ 3.00$. Special price for current issues to individual faculty members of supporting institutions and to individual members of the American Mathematical Society: $\$ 4.00$ per volume; single issues $\$ 1.50$. Back numbers are available.

Subscriptions, orders for back numbers, and changes of address should be sent to Pacific Journal of Mathematics, 103 Highland Boulevard, Berkeley, California, 94708.

PUBLISHED BY PACIFIC JOURNAL OF MATHEMATICS, A NON-PROFIT CORPORATION

Printed at Kokusai Bunken Insatsusha (International Academic Printing Co., Ltd.), 7-17. Fujimi 2-chome, Chiyoda-ku, Tokyo, Japan. 


\section{Pacific Journal of Mathematics}

Vol. 28, No. 3

May, 1969

Jon F. Carlson, Automorphisms of groups of similitudes over $F_{3} \ldots \ldots \ldots$

W. Wistar (William) Comfort, Neil Hindman and Stelios A. Negrepontis,

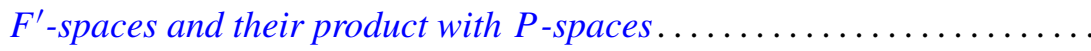

Archie Gail Gibson, Triples of operator-valued functions related to the unit

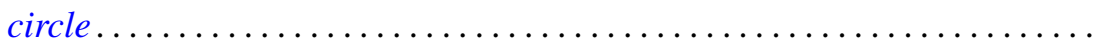

David Saul Gillman, Free curves in $E^{3}$

E. A. Heard and James Howard Wells, An interpolation problem for

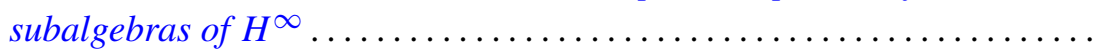

Albert Emerson Hurd, A uniqueness theorem for weak solutions of symmetric

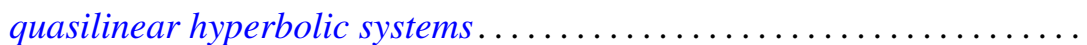

E. W. Johnson and J. P. Lediaev, Representable distributive Noether

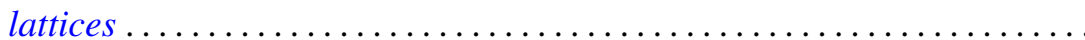

David G. Kendall, Incidence matrices, interval graphs and seriation in

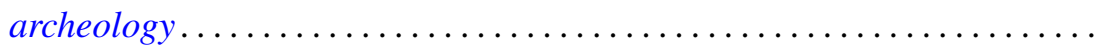
565

Robert Leroy Kruse, On the join of subnormal elements in a lattice ....... 571

D. B. Lahiri, Some restricted partition functions; Congruences modulo 3 .... 575

Norman D. Lane and Kamla Devi Singh, Strong cyclic, parabolic and conical differentiability........................................

William Franklin Lucas, Games with unique solutions that are

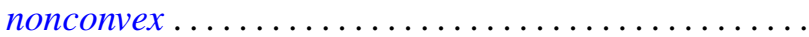

Eugene A. Maier, Representation of real numbers by generalized geometric series.

Daniel Paul Maki, A note on recursively defined orthogonal polynomials ...

Mark Mandelker, $F^{\prime}$-spaces and z-embedded subspaces ...

James R. McLaughlin and Justin Jesse Price, Comparison of Haar series with gaps with trigonometric series

Ernest A. Michael and A. H. Stone, Quotients of the space of irrationals ....

William H. Mills and Neal Zierler, On a conjecture of Golomb ...

J. N. Pandey, An extension of Haimo's form of Hankel convolutions ...

Terence John Reed, On the boundary correspondence of quasiconformal mappings of domains bounded by quasicircles...

Haskell Paul Rosenthal, A characterization of the linear sets satisfying Herz's criterion.

George Thomas Sallee, The maximal set of constant width in a lattice...

I. H. Sheth, On normaloid operators

James D. Stasheff, Torsion in BBSO ...

Billy Joe Thorne, A - P congruences on Baer semigroups.

Robert Breckenridge Warfield, Jr., Purity and algebraic compactness for

modules... 